\title{
Study on the Method of Calculating Temperature Rise of Transformer
}

\author{
Tao Li*, Xiaoping Du, Xuewu Sun, Yuanyuan Song \\ Shandong Electric Power Company, Jinan, Shandong, 250000, China
}

\begin{abstract}
The internal temperature of the transformer is a key parameter to measure the thermal state of the transformer. The service life of the transformer generally depends on the life of the insulating material, and high temperature is the main reason why cause insulation aging, this paper studies the temperature rise of transformer winding hot spot temperature for the key, using the neural network forecasting method, forecasts transformer winding hot spot temperature change rule, calculate the transformer internal temperature rise, provide the temperature of the scientific basis for the safe operation of the transformer.
\end{abstract}

\section{Introduction}

China's electricity demand is booming. As shown in table 1 , the statistical data of China electric power association show that China's installed capacity of full-caliber power generation and full-caliber power generation continue to increase year by year. It is estimated that China's installed power generation capacity will be 1.75 billion kilowatts by the end of 2017, and the country's electricity consumption will increase by about 3\% year-on-year. In addition to increasing construction investment, it is a feasible measure to use online monitoring of power equipment to improve resource utilization efficiency and improve refined operation and maintenance level of power equipment. The transformer is one of the most valuable core equipment in the power grid.

Table 1. Installation and power generation in China in recent years

\begin{tabular}{ccccc}
\hline Year & Installed Capacity & Growth Rate & Generating Capacity & Growth Rate \\
\hline 2014 & 11.44 & 8.33 & 4.98 & 5.21 \\
\hline 2015 & 12.47 & 9.01 & 5.35 & 7.43 \\
\hline 2016 & 13.60 & 9.06 & 5.55 & 3.74 \\
\hline 2017 & 15.10 & 11.03 & 5.60 & 0.90 \\
\hline 2018 & 16.50 & 9.27 & 5.99 & 6.96 \\
\hline
\end{tabular}

The temperature rise of power transformer is an important factor that affects its safe operation, and it is also one of the important concerns in the operation and maintenance of power grid. During the normal operation of the transformer, the safety and life loss of the oilpaper insulation system of the winding is mainly affected by the temperature of the hottest spot of the winding. Transformer winding hot spot temperature limits the maximum load capacity of transformer, is to determine the key factors of life of the transformer, the transformer top oil temperature and strong correlation to the winding hot spot temperature and easy to measure, both is one of the important parameters, measuring transformer internal thermal state to accurate calculation, transformer internal temperature rise in guaranteeing the safety on the basis of making full use of its capacity, increases the dynamic load capacity and push in the new transformer construction time.

At present, the methods of selecting the internal temperature of power transformers mainly include sensor measurement and theoretical model calculation. At present, direct measurement methods mainly include the use of electrical signal temperature sensor, optical signal humidity sensor, infrared temperature measuring device W. Transformers in service in the power grid are usually equipped with the top-layer oil temperature measuring device. Transformers in the $35 \mathrm{kV}$ voltage class are generally equipped with the thermistor temperature sensing device, and those in the low-voltage class are usually equipped with a thermometer holder on the box cover. Winding temperature is generally measured by optical signal temperature sensor. At present, the winding temperature mainly includes: optical fiber measurement method, stretch probe fiber measurement method and grating fiber temperature measurement method. The measurement accuracy of the method based on fiber optic temperature measurement is relatively accurate. However, according to the investigation of transformer manufacturers and power supply companies, power supply companies seldom use this method due to concerns about the insulation safety of transformer 
windings and the difficulty of increasing the operation and maintenance. The infrared temperature measurement method usually adopts the manual field hand-held infrared test gun or thermal imager to measure the surface temperature of the transformer. The field personnel qualitatively judge the thermal state of the transformer based on experience.

The theoretical calculation methods of internal humidity in transformers mainly include: numerical simulation model, semi-physical model based on simplified thermal balance relationship and data-driven mathematical model. Numerical simulation model mainly includes the model of the finite element method model of finite volume method and so on, the method based on the theory of heat transfer and fluid mechanics, a simplified $2 \mathrm{~d}$ or $\mathrm{H} \mathrm{d}$ model, and by solving the conservation of momentum, conservation of mass and energy conservation differential equations for solving the coupling field every bit of information, such as temperature, flow velocity. This kind of method needs the material and size of each key component of the transformer in detail, which is not good in universality. Moreover, it relies on professional calculation software, which is large in computation and poor in real-time, and is mostly used in transformer design. Half a physical model based on simplified thermal equilibrium relation mainly includes load guidelines recommend method and thermal load model method recommended a large number of recommended parameters are given, more suitable for the guide service running transformer generally run, but the model failed to adequately describe the nonlinear characteristics of the heat transfer process, the transient temperature rise process error is slightly larger. The model can well reflect the nonlinear characteristics of the transient process of temperature rise and the calculation accuracy is better.

\section{Factors affecting the internal temperature of the transformer}

Generally, the temperature rise of transformers is mainly caused by loss, the temperature rise of transformer core is mainly caused by no-load loss, and the temperature rise of transformer winding is mainly caused by load loss.

No-load loss refers to the ferromagnetic loss caused by magnetic flux on the transformer core, including hysteresis loss and eddy current loss, as well as the sum of copper wire loss generated by the transformer winding. Since the resistance of the transformer winding is usually small, the loss caused by it is far less than the ferromagnetic loss on the iron core. In order to simplify the calculation, the no-load loss is ignored as shown in equation (1).

$$
\mathrm{P}_{\mathrm{o}}=\mathrm{K}_{\mathrm{o}} \mathrm{GVp} \mathrm{p}_{\mathrm{o}}
$$

$\mathrm{P}_{\mathrm{o}}$ is no-load loss, $\mathrm{K}_{\mathrm{o}}$ is no-load loss additional coefficient, and $p_{o}$ is loss per unit weight of silicon steel sheet.
Load losses mainly include three types of losses :(a) losses caused by dc resistance;(b) additional losses caused by the winding;(c) stray losses of structural parts such as iron cores and clamps. In actual calculation, the calculation of load loss is shown in equation (2).

$$
\mathrm{P}_{L}=\mathrm{P}_{d c}+\mathrm{P}_{e c}+\mathrm{P}_{o s l}
$$

$\mathrm{P}_{d c}$ is the sum of resistance loss and current loss, $\mathrm{P}_{e c}$ is the eddy current loss of the winding, and $\mathrm{P}_{o s l}$ is the stray loss.

In the actual operation of the transformer, the temperature is increased due to the hysteresis loss, eddy current loss and the copper wire loss of the coil. Hysteresis and eddy current losses are essentially constant losses that cannot be reduced or eliminated in operation for a particular transformer, while copper wire losses vary with load. Of course, there are various reasons for the internal temperature rise of the transformer, and some of them are even difficult to determine. This is the temperature rise caused by internal reasons. According to the actual measured experimental data, the influence factors of transformer temperature rise are determined as active power $(\mathrm{P})$, reactive power $(\mathrm{Q})$, total voltage harmonic distortion rate $\left(V_{\text {thd }}\right)$, total current harmonic distortion rate $\left(I_{\text {thd }}\right)$, room temperature $\left(T_{\mathrm{i}}\right)$, and temperature of power distribution transformer in the first half hour (Tiron-1).

\section{The method of transformer temperature prediction based on neural network}

\subsection{Basic principle of neural network}

After the mathematical model of neuron is determined, the characteristics and abilities of a neural network mainly depend on the topology and learning method of the network. According to the topological structure of the connections between neurons, neural network can be divided into two categories: hierarchical network and interconnected network, and the structure of hierarchical network can be divided into monolayer, two-layer, and multi-layer according to the number of layers. All neurons are divided into several layers according to their functions, including the input layer, the hidden layer and the output layer Figure 1 shows a single-layer neural network model, including an input layer, a hidden layer and an output layer.

Input layer: the neurons at the node receive input patterns from the external environment and transmit them to the neurons at the connected hidden layer.

Hidden layer: the internal processing layer of the neural network. These neurons then form the middle layer inside the network. Since they do not directly deal with the external input and output, they are called hidden layer. The pattern transformation ability of neural 
network is mainly embodied in the hidden layer of neurons.
Output layer: an output used to generate a neural network.

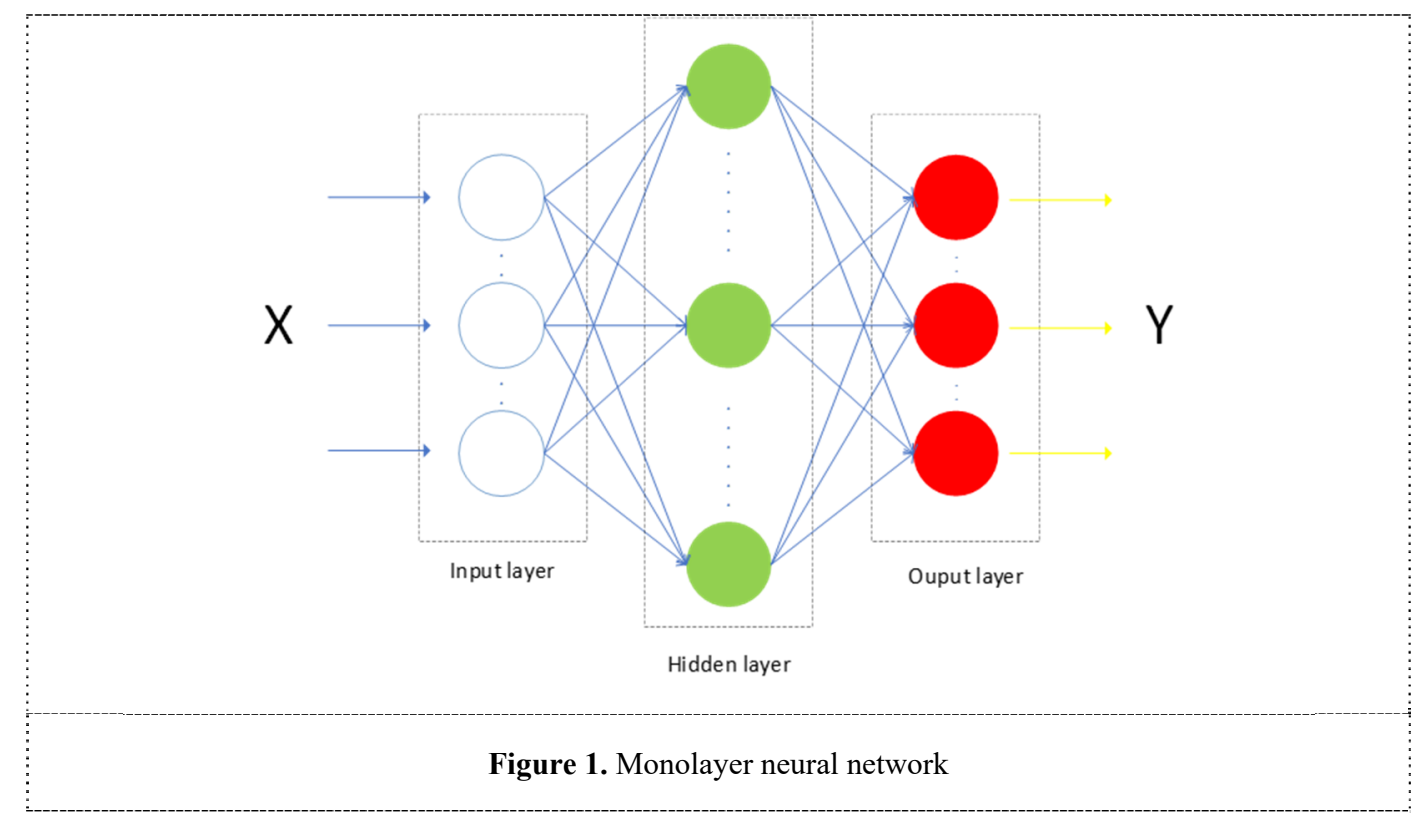

\subsection{Neural network design considering temperature rise factor of transformer}

(a) Selection of input layer and output layer

In the last chapter is on the basis of analysis of transformer internal heat generation principle, according to the actual measurement of experimental data, determine the factors which affect the transformer temperature rise for $\mathrm{P}, \mathrm{Q}, V_{\text {thd }}, I_{\text {thd }}, T_{\mathrm{i}}, T$ iron-1, respectively, In the case of standardization, fixed other variables, under the condition of single factor, respectively, to explore the $\mathrm{P}, \mathrm{Q}, V_{\text {thd }}, I_{\text {thd }}, T_{\mathrm{i}}$ effect of transformer temperature rise $\mathrm{T}$, through the test, educed the corresponding relationship each influence factor and temperature. Since the transformer is placed indoors, the influence of solar energy and wind speed can be ignored, and only the ambient temperature can be considered. Therefore, the three-layer BP neural network can be simplified into a single hidden neural network with six inputs and one output. The six inputs of the input layer are $\mathrm{P}, \mathrm{Q}, V_{\text {thd }}, I_{\text {thd }}, T_{\mathrm{i}}$, Tiron-1, respectively. The output of the network is the hot spot temperature value $\mathrm{T}$ of the transformer winding that needs to be obtained.

(b) Selection of hidden layer

The selection of the number of hidden layer units is a very complicated problem. The number of hidden layer units is directly related to the requirement of the problem, the number of input and output units. In the training process, this paper refers to the following empirical formula (3) and uses the heuristic method to determine the number of nodes in the hidden layer of the network.

$$
l=\sqrt{n+m}+\alpha
$$

Where, $l$ is the number of hidden layer elements, $n$ is the number of input layer elements, $m$ is the number of output layer elements, and the constant of interval $\alpha \in[1,10]$.

The number of nodes in each hidden layer was trained for 10 different times, and the number of nodes with the minimum average training error was taken as the optimal number of hidden layer nodes, so as to avoid the influence caused by randomness of initialization weight.

\section{Temperature prediction analysis based on monolayer neural network}

Based on active power ( $\mathrm{P}$ ), reactive power $(\mathrm{Q})$, total voltage harmonic distortion rate ( $\left.V_{\text {thd }}\right)$, total current harmonic distortion rate $\left(I_{\text {thd }}\right)$, room temperature $\left(T_{\mathrm{i}}\right)$, and temperature of power distribution transformer in the first half hour (Tiron-1) and so on six factors as six of the BP neural network input variables and output for the prediction of power distribution transformer temperature (T), and to predict the temperature rise of transformer. Its input-output model is shown in figure 2 . 


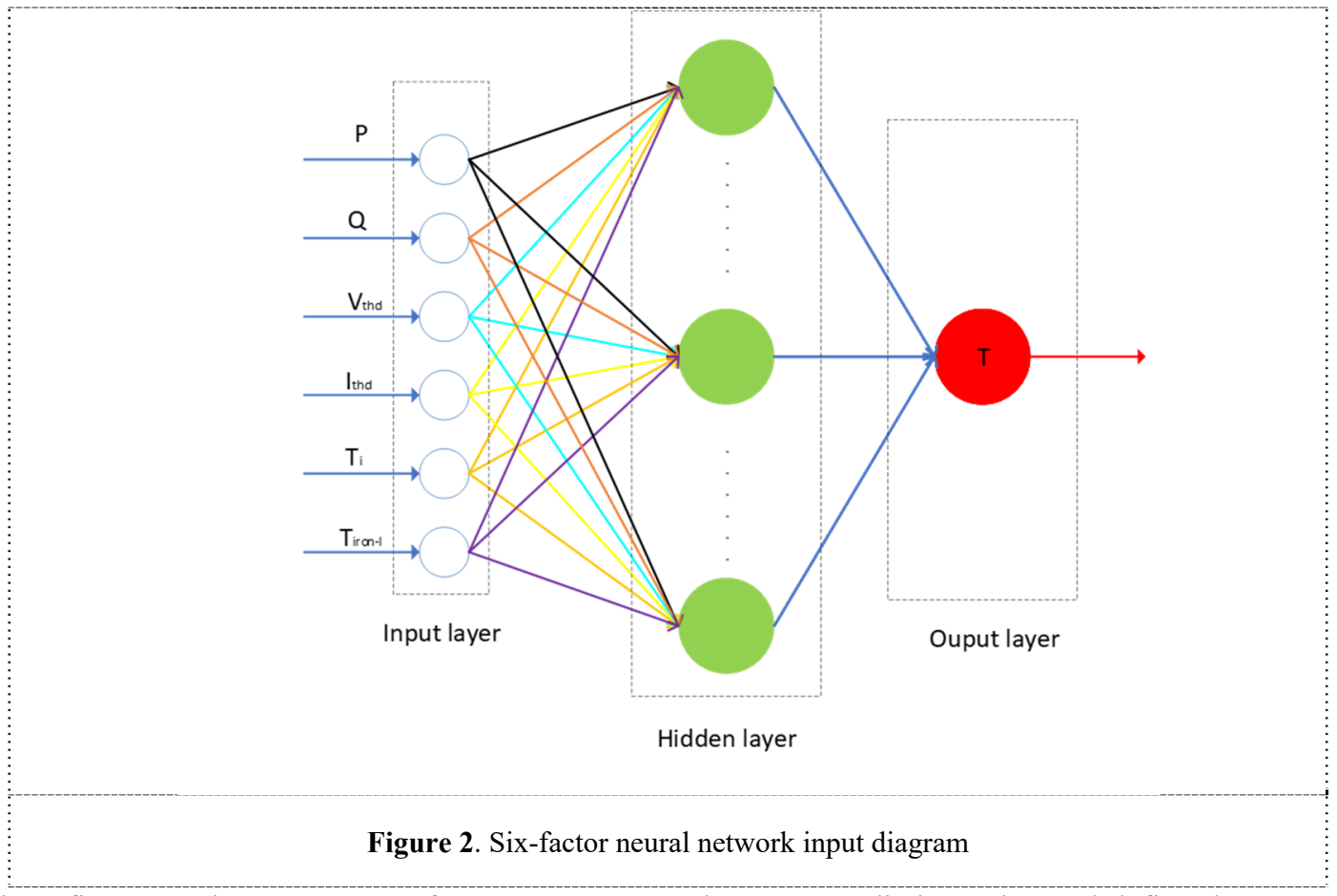

In table 2 first as a time sequence of measurement, and is used to determine the grouping of measurement data, the second as the actual measurement of transformer temperature value standard, the third as standard MAO and estimated values of calculation of neural network algorithm, the fourth as actual measured value and the algorithm of computing relative errors between predictive value and define the type of formula 4 shows, is

Percentage error $=$ (actual value - predicted value)/actual value $\times 100 \%$ (4)

The last row of the table calculates the average error percentage of the corresponding fourth column of the table.

Table 2. prediction of temperature rise in low-load area on working days

\begin{tabular}{cccc}
\hline Hour(h) & Actual Value (P.U.) & Experimental Value (P.U.) & Percent Error (\%) \\
\hline 0 & 0.8296 & 0.8197 & 1.193 \\
\hline 1 & 0.8333 & 0.8211 & 1.464 \\
\hline 2 & 0.8233 & 0.8244 & 0.134 \\
\hline 3 & 0.8202 & 0.8149 & 0.646 \\
\hline 4 & 0.8256 & 0.8173 & 1.005 \\
\hline 5 & 0.8195 & 0.8098 & 1.184 \\
\hline 6 & 0.8183 & 0.8134 & 0.599 \\
\hline 7 & 0.8190 & 0.8010 & 2.198 \\
\hline 20 & 0.8326 & 0.8145 & 2.174 \\
\hline 21 & 0.8323 & 0.8169 & 1.850 \\
\hline 22 & 0.8237 & 0.8170 & 0.813 \\
\hline 23 & 0.8301 & 0.8204 & 1.169 \\
\hline & Percent Mean Error $(\%)$ & & 1.233
\end{tabular}

Are shown in table 2 is a factor of six working days in low load prediction of temperature rise, the vast majority of the prediction error is around $1 \%$, as expected, the result of the error of the larger two percentage points respectively at about 7:00 in the morning, because most of the time on the early morning caused by load were launched, another is around $8 \mathrm{PM}$ tonight, because of work overtime at night time, load caused by suspended in succession. The average error in the low load area of the work day is acceptable.

Table 3. Forecast of temperature rise in stable area of working load

\begin{tabular}{cccc}
\hline Hour(h) & Actual Value (P.U.) & Experimental Value (P.U.) & Percent Error (\%) \\
\hline 8 & 0.8085 & 0.8104 & 0.240 \\
\hline 8.5 & 0.8152 & 0.8426 & 3.360 \\
\hline
\end{tabular}




\begin{tabular}{llll}
\hline 18 & 0.8286 & 0.8498 & 2.560 \\
\hline 19 & 0.8189 & 0.8275 & 1.050 \\
\hline & Percent Mean Error (\%) & & 1.694
\end{tabular}

Are shown in table 3 is a factor of six working days load fluctuation area prediction of temperature rise, error generally at about $1 \%$, the percentage error is bigger two points at 8:30, respectively for comparing concentrated load in the normal working hours in the morning start, transformer temperature fluctuation is bigger, the evening 18:00, so not to work overtime when normal work time, partial load caused by shut down one by one. The average error of temperature rise prediction in load changing zone is $1.694 \%$, and the overall prediction effect is in line with the expectation.

\section{Conclusion}

With six of transformer temperature rise influence factor, active power ( $\mathrm{P}$ ), reactive power ( $\mathrm{Q})$, total voltage harmonic distortion rate $\left(V_{\text {thd }}\right)$, total current harmonic distortion rate ( $\left.I_{\text {thd }}\right)$, room temperature $\left(T_{\mathrm{i}}\right)$, and temperature of power distribution transformer in the first half hour ( Tiron-1) as the neural network input variables, output to predict the temperature of the power distribution transformer, according to the change of the load, time-sharing subsection prediction of transformer temperature rise. Although this article made some achievements in study of transformer temperature rise, but it still has the following research space there are many factors that can affect transformer temperature rise, such as transformer environment of natural conditions, air humidity, ventilation effect, rainfall, factors such as solar energy will have an effect on the operation of the transformer and cooling, thus affecting the transformer temperature rise, this article is not to affect the transformer temperature rise of all factors into consideration.

\section{References}

1. Galdi, V.,Ippolito, L.,Piccolo, A.,Vaccaro, A., Electric Power Applications, IEE Proceedings .(2000) Neural diagnostic system for transformer thermal overload protection.,72-75.

2. Ippolito, L.,Siano, P. Generation, Transmission and Distribution (2004), Identification of TagakiSugeno-Kang fuzzy model for power transformers' predictive overload system., 1116-1118.

3. Xiaowu Qi,Kejun Li,Jingshan Wang,et al., Power and Energy Engineering Conference (APPEEC) (2016), Transformer top-oil temperature combination modeling based on thermal circuit and support vector machine.

4. W. N. Fu,P. Zhou,D. Lin,S. Stanton, and Z. J. Cendes., IEEE Transactions on Magnetics (2004). Modeling of Solid Conductors in Two-Dimensional
Transient Finite -Element Analysis and Its Application to Electric Machines.

5. Setayeshmehr, Alireza,Akbari, Alireza,Borsi, Hossein,Gockenbach, Ernst., IEEE Transactions on Dielectrics and Electrical Insulation (2006). On-line monitoring and diagnoses of power transformer bushings.

6. M.Fairouz,M.Yousof,Chandima Ekanayake,Tapan K.Saha., IEEE Transactions on Dielectrics and Electrical Insulation (2015). Examining the Ageing of Transformer Insulation Using FRA and FDS Techniques.

7. Bag heri M,Nade M S,BlackburnT,et al., IEEE Electrical Insulation Magazine (2013). Frequency response analysis and short-circuit impedance measurement in detection of winding deformation within power transformer.

8. M.Fairouz,M.Yousof,Chandima Ekanayake,Tapan K.Saha., IEEE Transactions on Dielectrics and Electrical Insulation (2015). Examining the Ageing of Transformer Insulation Using FRA and FDS Techniques. 